\title{
Absence of stimulus effects in dichotic singing*
}

\author{
BONNIE N. BARTHOLOMEUS \\ DONALD G. DOEHRING \\ and \\ STEVEN D. FREYGOOD \\ McGill University, Montreal, Quebec, Canada
}

\begin{abstract}
Dichotic melody recognition was assessed in four groups of 20 right-handed Ss, with the melodies performed on a violin or sung to vowels, consonant-vowel syllables, or digits. There were no significant differences between left- and right-ear scores on any of the four tasks. It was concluded tentatively that sung melodies involving varying degrees of verbalization show no greater trend for left-hemisphere specialization than do instrumental melodies.
\end{abstract}

Right-ear advantages on dichotic tasks involving speech sounds have generally been attributed to the encoded nature of the linguistic stimuli. However, because the required response usually involved spoken repetition, it is not clear whether the mere presence of encoded speech sounds in an auditory stimulus is a sufficient condition to produce a right-ear effect or whether it is also necessary that the experimental task require the decoding of linguistic information. Similar questions concerning the relationship between stimulus and task variables can be asked concerning the left-ear superiority found on tests of melody recognition (Kimura, 1964; Spellacy, 1970). Because the stimuli consisted only of instrumental music, it remains unclear whether the left-ear advantage is attributable to the absence of encoded speech sounds in the melodic stimuli or to the fact that the task of melody recognition does not require the decoding of linguistic information.

The need to specify the effects of stimulus and task variables in dichotic listening has recently been emphasized by Haggard (1971), who concluded that laterality effects are influenced to a greater degree by the nature of the perceptual task than by the acoustical attributes of the stimuli. If so, it can be hypothésized that when the perceptual task remains constant, laterality effects will not differ on tests which vary only according to the presence or absence of encoded linguistic information. In the present study, this hypothesis was tested by using melody recognition as the constant perceptual task and by varying systematically the linguistic content of the melodic stimuli by presenting the same melodies played on a violin and sung to vowels, to syllables, and to digits. In a

*The violin melodies were played by Daniel Ling. Thanks are also due Harriet Emerson and $R$ alph Booth for assistance in recording, Robert Rivard for testing, and Carolyn Cote for testing and data analysis. This research was supported by Grant MA-1652 from the Medical Research Council of Canada and National Health Grant 604-7-729 from the Canadian Department of National Health and Welfare. report that appeared while the present study was in progress, King \& Kimura (1972) found a significant left-ear advantage for hummed melodies and for other nonspeech vocalizations. Since the hummed melodies of King and Kimura correspond to our sung vowels, the present study should provide a further assessment of ear effects for the most nonverbal form of singing, along with a determination of whether singing is still processed in the same manner as instrumental music even when the linguistic content is systematically increased.

\section{METHOD}

The Ss were four groups of 10 male and 10 female university students. All were right-handed and had hearing threshold levels of $10 \mathrm{~dB}$ or less (ISO, 1964) at $500,1000,2000$, and $4000 \mathrm{~Hz}$. None had more than 3 years of formal music instruction, had previously listened to dichotic stimuli, or were familiar with the literature on dichotic listening. Stimuli were recorded at $71 / 2$ in./sec on an Ampex AG 500-2 tape recorder. Dichotic tapes were prepared by playing the master tapes from a Revox 77A tape recorder to the Ampex recorder for dubbing. All testing was done in a sound-treated room, with stimuli presented through TDH-39 earphones.

The third writer, a professional musician, composed 80 4-sec mclodies, divided into 20 sets of four. Within 10 of the sets, four melodies were written in one meter, and in the other 10 sets, two melodies were written in one meter and the other two in a different meter. No melody included notes lower than B below Middle $\mathrm{C}$ or higher than the second $\mathrm{F}$ above Middle $\mathrm{C}$.

Each of the four groups of Ss was given a different test of melody recognition, with each test including the same 20 sets of four melodies. For the group receiving instrumental melodies, all melodies were played on a violin. Each trial began with the dichotic melodies, followed in $5 \mathrm{sec}$ by binaural presentation of four recognition melodies separated by $4-s e c$ intervals. The $S$ had to identify the two melodies which had been presented dichotically. On half of the trials, the dichotic melodies and the four recognition melodies all had the same meter. On the remaining trials, one of the dichotic melodies and two recognition melodies were played in one meter, while the other member of the dichotic pair and the other two recognition melodies were played in a different meter. The remaining three groups received the same sets of melodies sung by the third writer, with one vowel repeated per melody (e.g., $/ \mathrm{i} / / \mathrm{i} / / \mathrm{i} / \ldots$. . one consonant-vowel syllable repeated per melody (e.g., $/ \mathrm{ba} / / \mathrm{ba} / / \mathrm{ba} / \ldots)$, and one set of three digits repeated per melody (e.g., 2,5,4 2,5,4), respectively. On the last test the four recognition melodies were sung to digits that had not been used for the dichotic melodies. Each S received only one of the four tests in a session of about $30 \mathrm{~min}$. Position of the headphones was reversed within each $S$ group so that half the $S$ s wore the right phone on the right ear.

\section{RESULTS}

Results of a 4 by 3 by 2 analysis of variance indicated no significant differences between tests, ears, or meters and no significant interactions among these variables. Percentages correct for the right and left ears, respectively, were $60 \%$ and $65 \%$ for instrumental melodies, $60 \%$ and $62 \%$ for sung vowels, $61 \%$ and $58 \%$ for vowel-consonant syllables, and $57 \%$ and $59 \%$ for 
sung digits. Within each group of 20 Ss there were at least 7 who showed no ear advantage or a right-ear advantage.

\section{DISCUSSION}

Since the recognition procedure, the number of trials, and the number of Ss per group were very similar to those employed by Kimura (1964) in demonstrating a significant left-ear advantage for melody recognition, the finding of no significant ear difference for instrumental melodies was unexpected. Both the results of the present study and those of Gordon (1970) might suggest that the use of a single instrument throughout the test would reduce ear differences, but this explanation conflicts with the significant left-ear effect found by Spellacy (1970) for dichotic violin melodies. It would seem more probable that the difference between Kimura's (1964) finding and that of the present study can be related to differences between the particular melodies used in the two studies. Granted this assumption, the results of the present study appear to confirm and extend those of King \& Kimura (1972) in the sense that relative ear superiority for sung and instrumental melodies was not significantly different, even when the sung melodies included syllables and digits. Thus, the absence of significant ear differences on all tests of melody recognition provides indirect support for the hypothesis that the relative performance of the two ears remains unchanged when the same perceptual task is performed in response to auditory stimuli differing only with respect to the presence or absence of encoded linguistic information. However, it is obvious that more research is needed to specify the effects of stimulus and task variables on the processing of melodic stimuli and human vocalizations.

\section{REFERENCES}

Gordon, $H$. W. Hemispheric asymmetries in the perception of musical chords. Cortex, 1970, 6, 387-398.

Haggard, M. P. Stimulus and task factors as determinants of ear advantages. Quarterly Journal of Experimental Psychology, $1971,23,168-177$

Kimura, D. Left-right differences in the perception of melodies. Quarterly Journal of Experimental Psychology, 1964, 16, 355-358

King, F. L., \& Kimura, D. Left-ear superiority in dichotic presentation of vocal nonverbal sounds. Canadian Journal of Psychology, 1972, 26, 111-116.

Spellacy, F. Lateral preferences in the identification of patterned stimuli. Journal of the Acoustical Society of America, 1970, 47, 574-578.

(Received for publication December 15, 1972.) 